\title{
The Ether and The Electrical Universe
}

\section{Paul TE Cusack*}

BScE, DULE, 23 Park Ave, Saint John, NB E2J 1R2, Canada

*Corresponding author: Paul TE Cusack, BScE, DULE, 1641 Sandy Point Rd., Saint John, NB E2K 5E, Canada

\begin{abstract}
Here we provide some basic calculations on the ellipsoid universe and the matter that makes it up called Ether. We see that, if we model the Ether as Teflon, a polymer, that certain constants drop out ion the calculations. This paper is to be read with the Astrotheology series by the same author, especially the paper The Ether: The Universal Material.
\end{abstract}

Keywords: Ether; Capacitance; Teflon; Polytetrafluoroethylene

\section{Introduction}

Using well established formula from the Physics of Electricity, we show that the ellipsoid universe can be modeled as a giant capacitor with the characteristics of Teflon [1,2]. We calculate the Mass Gap; Forces; Power and Capacitance.

$$
\begin{gathered}
\Delta V / V=\varepsilon_{0} E^{2} \\
19905=88.5418 E^{2} \\
E=149.15 \sim 150=\text { Mass gap } \\
F=\mu N \\
=[0.05](1)(1 / \sqrt{ } 2) \cos 60^{\circ} \\
=176.77 \\
\sim \sqrt{ } \pi=P \\
176.77 X \pi / 180^{\circ} \\
=3.06 \\
\sim c \\
E=F / Q=3.06 / 1.602=19101 \\
E=Q /\left[4 \pi r r^{2}\right] \bar{r} / r \\
19101=1602 /\left[4 \pi(88.5) r^{2}\right.
\end{gathered}
$$

$$
\begin{gathered}
\bar{r} / r=132.6 \\
r=s=d=4 / 3 \\
r^{2}=132.6 /(4 / 3) \\
r=3.15 \\
\sim \pi \\
V=i R \\
=4 / 3\left(10^{18}\right) \\
1.334\left(10^{18}\right) \\
P=i^{2} R \\
=(4 / 3)^{2}\left(10^{18}\right) \\
=1.777\left(10^{18}\right) \\
=\sqrt{ } \pi \\
P=V A \\
1.777=1.333 A \\
A=\frac{4}{3}
\end{gathered}
$$




$$
\begin{aligned}
& F=\mu N \\
& =\left(\cos 60^{\circ}\right)(0.05)\left(\frac{8}{3}\right) \\
& \text { S.F. }=8 / 3 \\
& =1.334 \\
& =s \\
& F=\mu N \\
& =\mu M a \\
& =(0.05)(\operatorname{Ln} 1.5)\left(\frac{1}{\sqrt{2}}\right) \\
& =1585 \\
& =1-\sin 1=\text { Moment } \\
& \text { Vol. }=\frac{4}{3} \pi R^{3} \\
& =\frac{4}{3} \pi(2 \pi)^{3} \\
& =0.14254 \\
& =\sin 59^{\circ}
\end{aligned}
$$

For half a sphere: $\mathrm{Vol}=7.127^{8}=6.65=\mathrm{G}$

$$
\text { Capacitance: }
$$

S.A. of ellipsoid:

$$
\begin{gathered}
=2 \pi\left(a^{2}+a b \cos \alpha / \sin \alpha\right) \\
a=1 \\
b=8 \\
\alpha=\frac{\pi}{2} \\
=9.836 \sim 10 \\
C=\varepsilon_{0} A \varepsilon_{R} / d=1 \\
4 / 3 \pi=8.854 \varepsilon_{R}(9.836) \\
\varepsilon_{R}=0.04809 \sim 0.05 \\
4 \pi /\left[3(88.54)(9836)=1 / \varepsilon_{R}\right. \\
\varepsilon_{R}=2.0788 @ 1 M H z .
\end{gathered}
$$

Teflon (Polytetrafluoroethylene) =Insulator

$$
\left(\mathrm{C}_{2} \mathrm{~F}_{4}\right)
$$

Density $\rho=2200 \mathrm{~kg} / \mathrm{m}^{3}$ (light weight concrete)

Young's modulus E=0.5GPa cf 0.4233

Bulk Resistance $=10^{18}$

Coefficient of Friction $=0.05-0.10=\mu$ (third Lowest $)$

Dielectric Strength $60 \mathrm{MV} / \mathrm{m}=\alpha$

Magnetism $10.28\left(10^{-6}\right)$

Note that Teflon is similar in structure to Beryllium Dichloride. The Ether mimics both Teflon and $\mathrm{BeCl}_{2}$. Note also that Teflon has the third lowest coefficient of friction. This property is conducive to the Super force generating space as we have shown above.

Permittivity of Free space

$$
\begin{gathered}
=4 \pi \\
=1.2566 \\
\sim E_{\text {min }} \\
H=I /[2 \pi R) \\
=4 / 3 /[(2 \pi)(1)] \\
=0.2122 \\
B=\mu H
\end{gathered}
$$$$
=(1.2566)(0.2122)=2.666=8 / 3=S . F .
$$

Magnetic Energy Density

$$
\begin{gathered}
W_{m}=1 / B H \\
=1 / 2(8 / 3)(0.2122)=2829=\operatorname{Ln} 126 \sim \operatorname{Ln} \rho
\end{gathered}
$$

(Figures 1\&2)

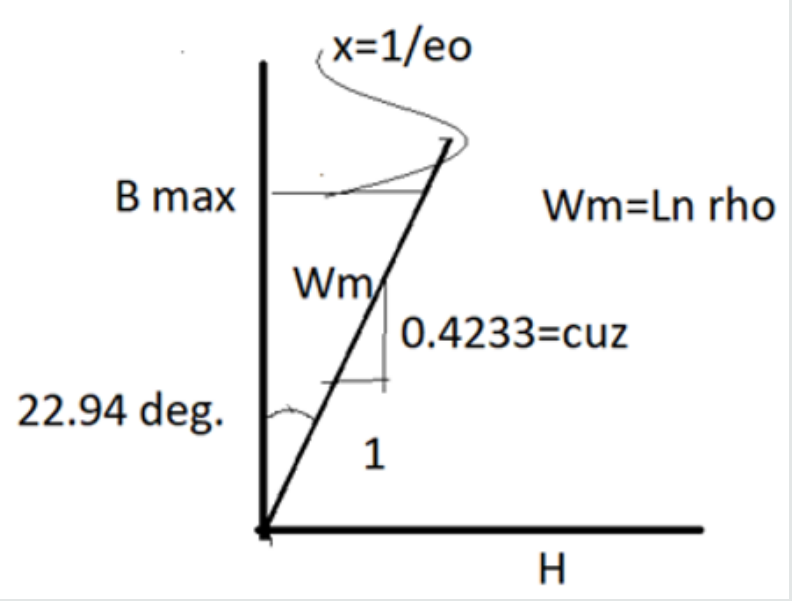

Figure 1: Magnetic Energy Density. 


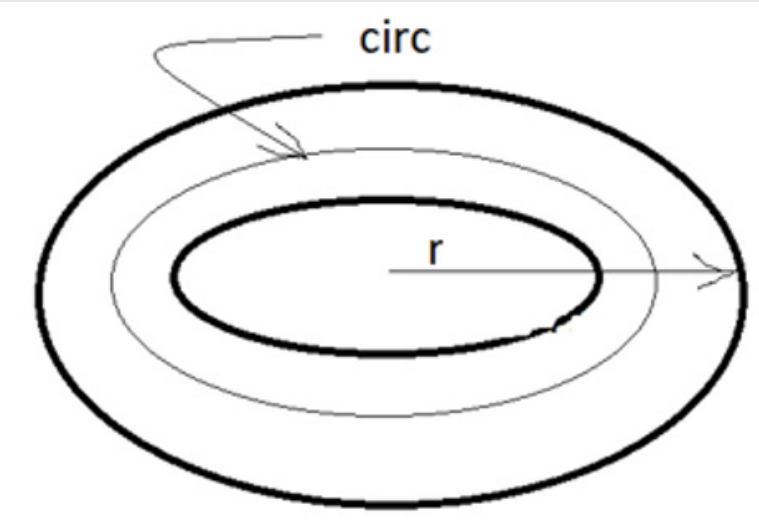

Figure 2: Torus Universe.

$$
\begin{gathered}
L=\mu l /[2 \pi][\operatorname{Ln}(2 l / r)-3 / 4] \\
2=1.2566 l /(2 \pi)[\operatorname{Ln} 4.482-3 / 4] \\
l=1 / 0.75 \\
=4 / 3 \\
i \\
\operatorname{Ln}(4.482)=\operatorname{Ln}(2 l / r) \\
1.5=2(4 / 3) / r \\
r=1.777=s q r t P i \\
\text { Power } P P=i^{\wedge} 2 R \\
1.777=(4 / 3)^{\wedge} 2 R \\
R=3.159 \sim P i=C a p .
\end{gathered}
$$

For a loop (Torus Universe)

$$
\begin{aligned}
& l=4 / 3 \\
& r=1.777
\end{aligned}
$$

Circumference $=2 \pi R$

$$
\begin{gathered}
L=2 \pi(1.777)=1.116 \\
=1 / c^{\wedge} 2
\end{gathered}
$$

Short Circuit Power:

$$
\begin{gathered}
P_{\text {max }}=1 / 4 V_{Q}^{2 / R} a \\
P=V \times A \\
=1.25 \times 4 / 3 \times 4)=0.666=G \\
=V_{Q}{ }^{2 /} R_{a}=P_{k} \\
R_{\text {TOT }}=R+1 / C-L
\end{gathered}
$$

$$
\begin{gathered}
=0.4233+1 / \pi-2=1.2587 \sim 4 \pi \\
=\mu \\
c=1 /[\sqrt{\varepsilon \cdot \mu}] \\
=1 /[\sqrt{ } 0.8854 \cdot 4 \pi] 2.9979
\end{gathered}
$$

Speed of electromagnetic radiation

$$
\begin{aligned}
& d I(t) / d t=-1 / \tau(t)+V_{0} / L \\
& \tau=L / R=2 / 0.4233=4.724 \\
& d I(t) / d t=-4.724(4 / 3)+(-1.25) / 2) \\
& =-6.3-0.625=-6.925 \\
& V_{L}(t)=V_{0} e^{-1 / \tau} \\
& =-1.25 e^{\frac{-1}{4.724}}=-1.25(0.80922)=-1.0115 \\
& I(t)=I_{0}\left(1-e^{-1 / \tau}\right) \\
& =4 / 3((1-0.80922)=0.143733 \\
& V(t) / I /(t)=-6.957 \sim i(t) / d t \\
& V=I R \\
& V=1 / I \\
& 1 / I=I R \\
& 1=I^{2} R=\text { Power } \\
& f=1 / \pi \cdot 1 /(8 \pi) \\
& \omega=2 \pi f \\
& 1 / 4 \cdot 1 / \pi \\
& =0.25
\end{aligned}
$$

Period T

$$
i(t)=i^{\wedge} \sin (\omega t+\varphi)
$$$$
4 / 3 \sin \left(0.25(0.396)+60^{\circ}\right)
$$$$
4 / 3(0.99+1.047)
$$$$
4 / 3(0.99+1.047)
$$$$
=2.71626
$$$$
=e^{0.999}
$$$$
=e^{\wedge}-t
$$$$
t=-0.999
$$$$
T=
$$ 


$$
\begin{gathered}
V=\hat{v} \sin (\omega t+\varphi) \\
=1.25(203.7)=254.625 \\
=\text { Period } T=1 / t \\
E \\
E=-1.0115 \\
t=-0.999 \\
-0.999+1.0115=d M / d t \\
0.99+1.0115=-2=d M / d t \\
\int d M / d t=\int(E+t) d M \\
M=(E+t) M \\
1=(1 / t+t) ; t=[1+t] / t ; t^{2}=1+t ; t^{2}-t-1=0 \\
\text { Golden Mean Parabola } \\
\text { Euler's Formula } \\
e^{i \varphi}=\cos \varphi+i \sin \varphi
\end{gathered}
$$

We know that $i=j=\sqrt{-1}=-0.618$ The Conjugate of the Golden Mean.

Let $\varphi=\pi / 4$ so that :

$$
\sin \varphi=\cos \varphi=1 / \sqrt{2}
$$

$$
e^{-0.618 \varphi}=\cos (\pi / 4)+(-0.618) \sin (\pi / 4)
$$

$$
e^{-0.618 \varphi}=2.701=v=\text { Poisson's Ratio }
$$

Take the Ln of both sides:

$$
\begin{gathered}
-0.618 \varphi=\operatorname{Ln} 0.2701 \\
\varphi=0.2118 \\
\text { Now, } \\
v(t)=v^{\wedge} e i(\omega t+\varphi) \\
=v^{\wedge} e-0.618((1)(1)+0.2118) \\
=-1.25(0.4727) \\
=-59089 \\
i(t)=i^{\wedge} e i(\omega t+\varphi) \\
=4 / 3(0.5011) \\
=0.668 \\
\sim G
\end{gathered}
$$

Resistance

$$
Z=v / i
$$

$=0.59089 / 0.668=0.884565$

$\sim \varepsilon_{0}=$ Permittivity of Free Space

\section{Conclusion}

We see that the field of electricity provides basic formula that can be used to model the ellipsoid universe.

\section{References}

1. Cusack PTE (2016) The Ether: The Universal Material. Fluid Mech Open Acc 3: 132.

2. Benenson, W, Harris JW, Stöcker H, Lutz H (2012) Handbook of Physics. Springer New York, USA.

\section{(C) \\ This work is licensed under Creative Commons Attribution 4.0 License}

To Submit Your Article Click Here:

Submit Article

DOI: 10.32474 /MAMS.2020.02.000143

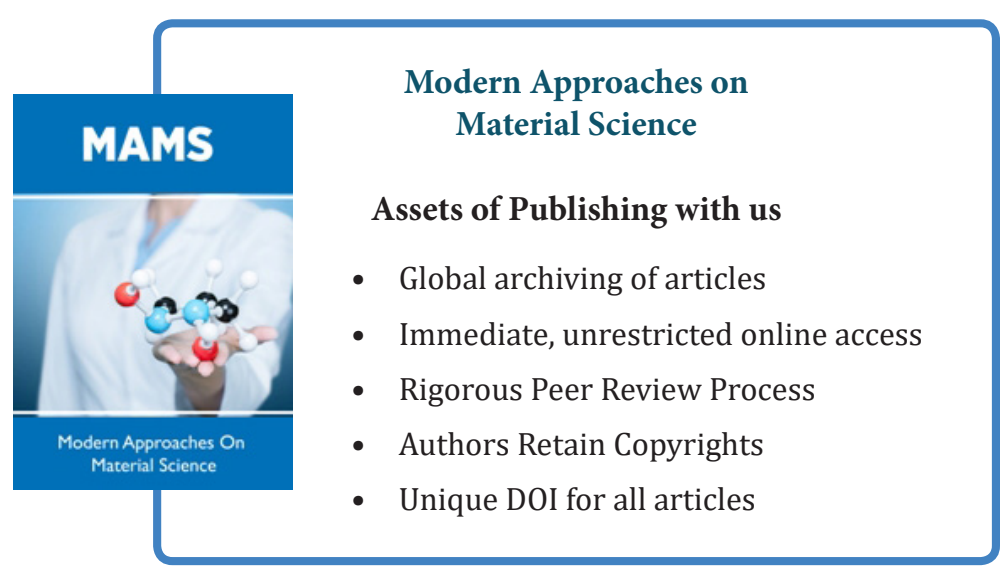

